\title{
PIK3CA and PTEN Genes Expressions in Breast Cancer
}

\author{
Nabil Hadi Alowiri ${ }^{1 *}$, Shaden Muawia Hanafy ${ }^{1}$, Reham Abdel Haleem², Ahmed \\ Abdellatif $^{3}$
}

\begin{abstract}
Background: The phosphatidylinositol-3 kinase (PI3K) intracellular signaling pathway plays an important role in breast cancer. The current study aimed to evaluate the expressions of two main regulators of PI3K pathway; phosphatidylinositol-3- kinase catalytic subunit alpha as activator (PIK3CA), and phosphatase and tensin-homolog as inhibitor (PTEN), in breast carcinoma tissue, and compare with their expressions in adjacent normal breast tissue. Methods: A total of fifty female patients with breast carcinoma from surgical oncology unit of Alexandria-Main University Hospital were included in this study. The Quantitative Real Time PCR was used to quantify expressions of PIK3CA and PTEN. Results: PIK3CA $m R N A$ expression was significantly increased in breast cancer tissues compared to normal breast tissues $(\mathrm{P}<0.001, \mathrm{Z}=5.700)$, also $P T E N m R N A$ expression was significantly higher in breast carcinoma tissue compared to normal breast tissue $(\mathrm{P}<0.001, \mathrm{Z}=5.362)$. Conclusion: Increased the expressions of $P I K 3 C A$ and PTEN $m R N A$ in breast cancer tissue compared to normal breast tissue.
\end{abstract}

Keywords: PIK3CA-PTEN- expressions- breast cancer

Asian Pac J Cancer Prev, 20 (9), 2841-2846

\section{Introduction}

Breast cancer (BC) is a major lethal public health problem in women worldwide, it is the second leading cause of cancer death after lung cancer in women with more than one million new cases are diagnosed every year (Siegel et al., 2016; Segal et al., 2018). In 2018, one in eight women in the USA had a lifetime risk of developing breast cancer (Segal et al., 2018), while in Egypt according to a previous a study the breast cancer accounts for $15.4 \%$ of all cases of cancer but in female it accounts for $32.04 \%$ of female cases of cancer (Ibrahim et al., 2014). The molecular biomarkers, and their correlation with pathological parameters are very important for diagnosis, prognosis, predictive utility, management, and prevention of breast cancer (Patani et al., 2013).

The phosphatidylinositol-3-kinase (PI3K) pathway is an important signaling pathway in cells and is involved in essential cellular functions such as metabolism, proliferation, survival, motility, and growth and plays a main role in development and progression of breast cancer (Engelman et al., 2006; Boyault et al., 2012).

Based on structure, regulation, and substrate specificity, the PI3K family is classified into three classes, class I, class II, and class III (Leevers et al., 1999). Class I is divided to IA and IB, class IA PI3K is composed of two subunits: A regulatory subunit $(85 \mathrm{kDa})$, and a catalytic subunit $(110 \mathrm{kDa})$ of which contain four isoforms $(\alpha, \beta, \gamma$, and $\delta$ ) that can be activated by tyrosine kinase receptors (Bellacosa et al., 2005). The $\alpha$ isoform of catalytic subunit (PIK3CA) is encoded by PIK3CA gene which is located on chromosome 3 (3q26.32). It has been found amplified or mutated in several cancer types including breast, ovary, colon, liver, stomach, brain, and lung (Samuels et al., 2004; Levine et al., 2005). When PI3K activated converts the phosphatidylinositol -4,5-bisphosphate (PIP2) to phosphatidylinositol -3,4,5-trisphosphate (PIP3) that leads to downstream signaling through a $\mathrm{PI} 3 \mathrm{~K} / \mathrm{AKT} / \mathrm{mTOR}$ pathway (Wang et al., 2011).

On the other hand, the PI3K activation is inhibited by phosphatase and tensin-homolog (PTEN) which converts PIP3 to PIP2 (Wang et al., 2011). PTEN protein is involved in DNA repair, apoptosis, cell cycle progression, and proliferation, and is encoded by PTEN gene, it is a tumor suppressor gene located on chromosome 10 (10q23). Changes of expressions is the most common alteration of PTEN that may leads to tumor cell growth, escape from cell cycle arrest, and apoptosis signals (Leslie and Downes 2004; Song et al., 2012).

The PI3K/AKT/mTOR pathway has a major role in the resistance to anticancer drugs and in the response to treatment (Leslie and Downes, 2004). In the recent strategies of cancer treatment and depending on PI3K pathway can be use the PI3K inhibitors to cancer treatment 
which is used alone or combined with other strategies of cancer therapy (Fruman and Rommel, 2014, Arnedos et al. 2015).

The present study aimed to assess the mRNA expression of PIK3CA and PTEN genes in the breast carcinoma tissue compared to their expression in the normal breast tissue, and evaluated the correlation of their expression with clinicopathological parameters in patients with $\mathrm{BC}$.

\section{Materials and Methods}

\section{Patients and samples}

The current study was conducted on fifty female patients with breast cancer selected from surgical oncology unit of Alexandria-Main University Hospital, Alexandria, Egypt. The study was previously approved by the Ethical Committee of the Genetic Engineering and Biotechnology Research Institute, University of Sadat City. Fresh tissue of breast carcinoma and adjacent normal tissue were collected from each patient during the excision surgery and immediately stored at $-80^{\circ} \mathrm{C}$. Discrimination between breast cancer tissues and the adjacent normal tissues was made by histopathological examinations.

Clinical parameters were collected for all patients, including age, tumor size, type, grade, lymph node metastasis, and immunohistochemical situation of estrogen receptor (ER), progesterone receptor (PR), and human epidermal growth factor receptor 2 (HER-2).

\section{RNA extraction and $c D N A$ synthesis}

The RNA was extracted using PureLink ${ }^{\circledR}$ RNA Mini Kits for purification of total RNA (Invitrogen TM, Carlsbad, CA 92008, USA). Catalog Number (12183018A) according to the manufacture instructions. Fifty mg of frozen tissue were used to extract the total RNA, the tissues were homogenized and grinded by a Tissue Lyser LT (Qiagen, Germany). It was operated for 5 min at 50 Hertz. The quantity and purity of extracted RNA was determined by NanoDrop ND-2000/2000c spectrophotometer (ThermoScientific, USA).

High-Capacity cDNA Reverse Transcription Kit was used for cDNA synthesis (Applied Biosystems, Carlsbad, CA 92008, USA) according to the manufacture instructions.

\section{Quantitative expression examination}

Quantitative Real Time PCR (qPCR) was used to determine the expression levels of PIK3CA and PTEN genes. GAPDH was used as endogenous reference for both genes. The relative expression was determined using Applied Biosystem 7500 Fast Dx Real- Time PCR System (Applied Biosystem, USA).

GAPDH, as the appropriate housekeeping gene, was used to control for variations in RNA concentration and integrity. The qPCR was performed using the Maxima SYBR Green qPCR Master Mix (2X) kit (Ferments, Thermo Fisher Scientific Inc. Catalog Number K0251). Specific primers were used to quantify $P I K 3 C A$, PTEN, and GADPH genes (Ferments, Thermo Fisher Scientific Inc. USA). The sequences of primers were:
PIK3CA Forward: GGCCACTGTGGTTGAATTGGGA, Reverse: AGTGCAC-CTTTCAAGCCGCC, PTEN Forward: TGGGCCCTGTACCATCCCAAGT, Reverse: TGTGGCAACCACAGCCATCGT, GAPDH Forward: AAGGTCGGAGTCAACGG-ATTTG, and Reverse: GCCATGGGTGGAATCATATTGG. The amplicons size of the amplified products were: PIK3CA $250 \mathrm{pb}$, PTEN $445 \mathrm{pb}$, and GAPDH $150 \mathrm{pb}$. Real-Time PCR System was programmed as follows: one cycle at $95^{\circ} \mathrm{C}$ for $10 \mathrm{~min}, 40$ cycles at $95^{\circ} \mathrm{C}$ for $15 \mathrm{sec}$, and $60^{\circ} \mathrm{C}$ for $60 \mathrm{sec}$. Melting curve analysis was used to determine the specificity of the PCR products after each run.

\section{Statistical analysis}

Data were fed to the computer and analyzed using IBM SPSS software package version 20.0. (Armonk, NY: IBM Corp) Qualitative data were described using number and percent. Quantitative data were described using range (minimum and maximum), mean, standard deviation and median. The t-test $(\mathrm{t})$, chi-square test $\left(\chi^{2}\right)$, Wilcoxon signed ranks test $(Z)$, and Spearman coefficient (rs) were performed for statistical examination.

\section{Results}

The present study included fifty female patients with breast cancer, and they had a mean age 54 age years (range: 19-80 years). The clinicopathological features of all patients were listed in Table 2 .

When we compared PIK3CA mRNA expression in breast carcinoma tissues with its expression in normal breast tissues observed significantly higher expression in carcinoma tissues than in normal tissues $(\mathrm{P}<0.001$, $\mathrm{Z}=5.700$ ). The median value for $P I K 3 C A / G A D P H$ ratio in breast carcinoma tissues was 42.35 compared to 1.12 PIK3CA/GADPH in normal breast tissues. High PIK3CA expression (above median level 42.35) was in 25 patients (50\%), while the low PIK3CA expression $(\leq 42.35)$ was in $50 \%$ of patients ( 25 patients). In breast tumor tissue observed increase the PIK3CA $m R N A$ expression in 47 patients out of 50 (94\%). Table1, Figure 1a.

Also, PTEN $m R N A$ expression was significantly higher in carcinoma tissues than in normal breast tissues $(\mathrm{P}<0.001, \mathrm{Z}=5.362)$. The median value for $P T E N / G A D P H$ ratio in breast carcinoma tissues was 13.76 compared to 2.42 PTEN/GADPH in normal breast tissues. High PTEN

Table 1. Descriptive Analysis of the Studied Cases According to PIK3CA and PTEN Expression $(\mathrm{n}=50)$

\begin{tabular}{|c|c|c|c|c|}
\hline & Tumor Tissues & Normal Tissues & $\mathrm{Z}$ & $\mathrm{p}$ \\
\hline \multicolumn{3}{|c|}{$P I K 3 C A$ expression } & $5.700 *$ & $<0.001 *$ \\
\hline Min. - Max. & $5.98-2288.2$ & $0.03-335.5$ & & \\
\hline Mean \pm SD & $131.4 \pm 328.6$ & $11.45 \pm 48.12$ & & \\
\hline Median & 42.35 & 1.12 & & \\
\hline \multicolumn{5}{|c|}{ PTEN expression } \\
\hline Min. - Max. & $0.02-2957.2$ & $0.0-16.34$ & & \\
\hline Mean $\pm \mathrm{SD}$ & $281.3 \pm 554.7$ & $4.16 \pm 4.32$ & $5.362 *$ & $<0.001 *$ \\
\hline Median & 13.76 & 2.42 & & \\
\hline
\end{tabular}

$\mathrm{Z}, \mathrm{p}, \mathrm{p}$ value for Wilcoxon signed ranks test for comparing between pathogenic and normal; *, Statistically significant at $\mathrm{p} \leq 0.05$ 
DOI:10.31557/APJCP.2019.20.9.2841

PIK3CA and PTEN Genes Expressions in Breast Cancer

Table 2. Relations between PIK3CA and PTEN Genes Expressions and Clinicopathological Features of BC

\begin{tabular}{|c|c|c|c|c|c|c|c|c|}
\hline & \multicolumn{2}{|c|}{$P I K 3 C A$ expression $(50)$} & \multicolumn{2}{|c|}{ significance } & \multicolumn{2}{|c|}{ PTEN expression (50) } & \multicolumn{2}{|c|}{ significance } \\
\hline & Low PIK3CA & High $P I K 3 C A$ & $\mathrm{X}^{2}$ & $P$ value & Low PTEN & High PTEN & $\mathrm{X}^{2}$ & $\mathrm{P}$ value \\
\hline Number, N (\%) & $25(50 \%)$ & $25(50 \%)$ & & & $25(50 \%)$ & $25(50 \%)$ & & \\
\hline Age, median (range) & $53(19-72)$ & $55(36-80)$ & & 0.421 & $51(19-73)$ & $55(35-80)$ & & 0.16 \\
\hline Tumor size, mm, median (range) & $17(7.8-18)$ & $19(8.3-20.4)$ & & 0.291 & $17(9.6-40)$ & $19(5-33)$ & & 0.289 \\
\hline \multicolumn{9}{|l|}{ Histology, N (\%) } \\
\hline Ductal & $23(92)$ & $22(88)$ & 0.222 & 1 & $23(92)$ & $22(88)$ & 0.222 & 1 \\
\hline Lobular & $2(8)$ & $3(12)$ & & & $2(8)$ & $3(12)$ & & \\
\hline \multicolumn{9}{|l|}{ Tumor grade, N (\%) } \\
\hline I & $4(16)$ & $2(8)$ & & & $1(4)$ & $5(20)$ & & \\
\hline II & $9(36)$ & $16(64)$ & 3.864 & 0.147 & $14(56)$ & $11(44)$ & 2.924 & 0.275 \\
\hline III & $12(48)$ & $7(28)$ & & & $10(36)$ & $9(36)$ & & \\
\hline \multicolumn{9}{|l|}{ ER status, N (\%) } \\
\hline Positive & $7(28)$ & $8(32)$ & 0.095 & 0.758 & $6(24)$ & $9(36)$ & 0.857 & 0.355 \\
\hline Negative & $18(72)$ & $17(68)$ & & & $19(76)$ & $16(64)$ & & \\
\hline \multicolumn{9}{|l|}{ PR status, N (\%) } \\
\hline Positive & $11(44)$ & $14(56)$ & 0.72 & 0.396 & $10(40)$ & $15(60)$ & 2 & 0.157 \\
\hline Negative & $14(56)$ & $11(44)$ & & & $15(60)$ & $10(40)$ & & \\
\hline \multicolumn{9}{|l|}{ HER2 status, N (\%) } \\
\hline $1+$ & $11(44)$ & $10(40)$ & & & $9(36)$ & $12(48)$ & & \\
\hline $2+$ & $7(28)$ & $7(28)$ & 0.114 & 0.944 & $9(36)$ & $5(20)$ & 1.638 & 0.441 \\
\hline $3+$ & $7(28)$ & $8(32)$ & & & $7(28)$ & $8(320$ & & \\
\hline \multicolumn{9}{|l|}{ Lymph node status, N (\%) } \\
\hline Metastasis & $6(24)$ & $8(32)$ & 0.397 & 0.529 & $8(32)$ & $6(24)$ & 0.397 & 0.529 \\
\hline No metastasis & $19(76)$ & $17(68)$ & & & $17(68)$ & $19(76)$ & & \\
\hline
\end{tabular}

High PIKECA expression $>42.35$ (median); low PIKECA expression $\leq 42.35$; High PTEN expression $>13.76$ (median); low PTEN expression $\leq 13.76$.

expression (above median level 13.76) was in 25 patients $(50 \%)$, and the low PTEN expression $(\leq 13.76)$ was in 25 patients $(50 \%)$. In the PTEN gene, only $8(16 \%)$ out of 50 carcinoma samples had decreased gene expression, while the $42(84 \%)$ had increased expression. Table 1, Figure $1 \mathrm{~b}$.

Clinicopathological characteristics did not show any significant association with PIK3CA and PTEN genes expressions ( $\mathrm{P}>0.05)$, Table 2 .

PIK3CA expression in breast carcinoma did not show

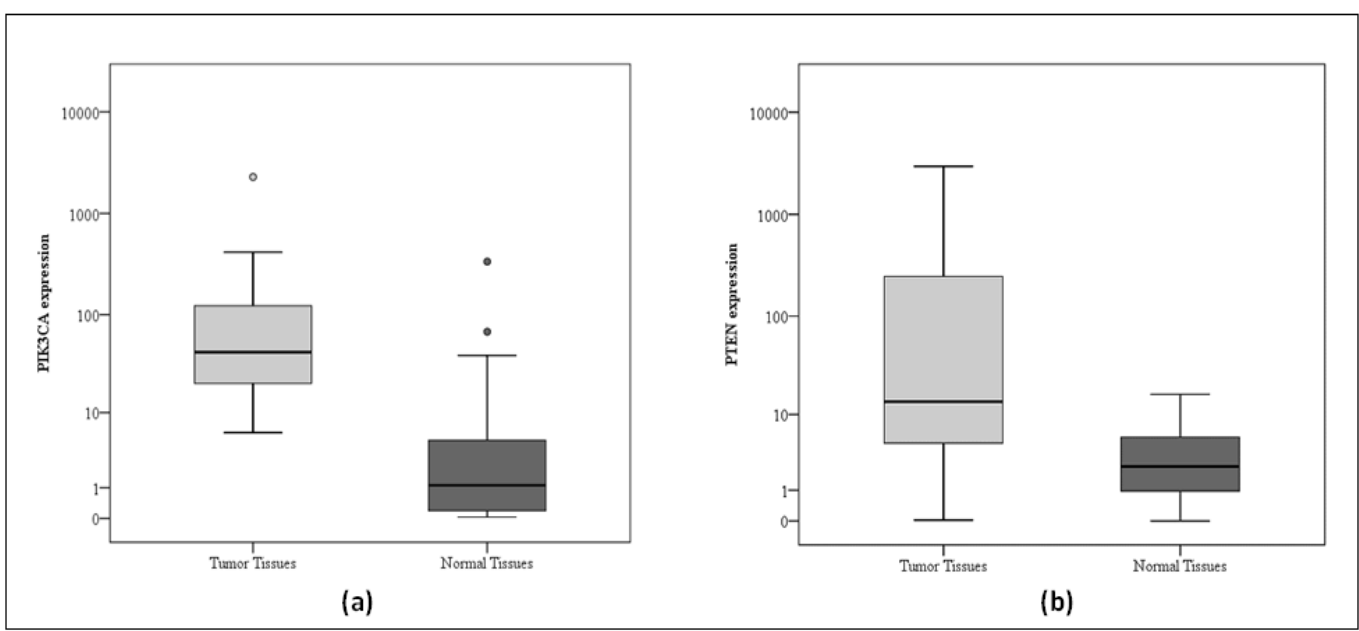

Figure 1. Descriptive Analysis of the Studied Cases According to PIK3CA (a) and PTEN (b) Expressions (n=50)

a significantly positive correlation with $P T E N$ expression $(\mathrm{rs}=-0.05, \mathrm{P}=0.723)$, Table 3 .

Table 3. Correlation between PIK3CA Expression and $P T E N$ Expression in Breast Carcinoma

\begin{tabular}{llc}
\hline PTEN expression & \multicolumn{2}{l}{ PIK3CA expression } \\
& $\mathrm{r}_{\mathrm{s}}$ & $\mathrm{p}$ \\
\hline & -0.051 & 0.723 \\
\hline $\mathrm{r}_{\mathrm{s}}$, Spearman coefficient & &
\end{tabular}




\section{Discussion}

Breast cancer is the most common cancer among women, and the second most frequent cancer worldwide (Siegel et al., 2016; Segal et al., 2018). PI3K pathway play a crucial role in development and progression of cancers mostly breast cancer (Boyault et al., 2012), in this study we focused on the main regulators of the $P I 3 K$ pathway (PIK3CA which acts as PI3K activator and PTEN acts as $P I 3 K$ inhibitor and tumor suppressor), and were examined to test whether an increased PIK3CA expression and decreased $P T E N$ expression in breast carcinoma and normal breast tissue.

Most previous studies to assess genes expressions have used immunohistochemistry (IHC) methods that are based on detect proteins molecules by antibodies, (Tsutsui et al., 2005; Aleskandarany et al., 2010) and there were some studies used quantitative method by real time PCR to assess the mRNA expressions of target genes. In this study used also the real time qPCR to detect the mRNA expressions of PIK3CA and PTEN genes because this method is a highly sensitive, quantitative, reproducible and accurate (Vanden et al., 2005; Salmani et al., 2018).

In the current study, we measured the mRNA expression of PIK3CA and PTEN genes in breast cancer tissue and compared them with their expression in normal breast tissue, and according to data of this study the PIKECA expression showed significantly higher in breast carcinoma tissue than in normal breast tissue, where the median value of $P I K 3 C A$ expression in breast carcinoma was 42.35 whereas in normal tissue was 1.12 $(\mathrm{P}<0.001)$. This result may support the hypothesis of the PI3K pathway play a crucial role in development and progression of breast cancer (Boyault et al., 2012).

To our knowledge, there is not many studies about $P I K 3 C A$ gene expression in breast cancer, most studies focused on PIK3CA mutations in breast cancer. PIK3CA expression data in the current study similar to data in the Palimaru et al., (2013) study that showed significantly higher $P I K 3 C A$ expression in breast carcinoma than in normal breast tissue $\left(\mathrm{p}=2 \times 10^{-11}\right), P I K 3 C A$ expression was increased in breast carcinoma in $76 \%$ of patients $(114 / 149)$ whereas in our study the $P I K 3 C A$ expression was increased in breast carcinoma in $96 \%$ of patients $(47 / 50)$. Also, there was a previous report at 2010 (Aleskandarany et al., 2010) showed PIK3CA high expression in breast tumor tissue with IHC.

We compared the PIK3CA mRNA expression with clinicopathological parameters (age, tumor size, tumor histology, tumor grade, ER, $P R, H E R 2$, and lymph node status) and we did not observe significant association between PIK3CA expression with any clinicopathological parameter $(\mathrm{P}>0.05)$, that is corresponds to the results of the Palimaru et al. study (Palimarun et al., 2013), and conflict with Aleskandarany et al., (2010) study, which reported significant associated between increased PIK3CA expression with histologic tumor type, higher tumor grade, larger tumor size, negative hormone receptors $(A R$ and $P R$ ), positive $H E R 2$, and lymph node metastases, and showed no significant association with age.

The PTEN gene encodes a lipid phosphatase that acts as an inhibitor of $P I 3 K$ and influences on the $A K T$ pathway regulations. Decreased or loss of PTEN expression leads to activated and increased levels of the $A K T$, thus promoting cell cycle progression, cell survival, proliferation, and migration, this is a major cause of tumors creation (Majumder and Sellers, 2005). The results of PTEN gene expression in this study were contrary to expected, the expression was significantly higher in carcinoma tissue than in normal breast tissue $(\mathrm{P}<0.001, \mathrm{Z}=5.362)$ where the median value of PTEN expression in breast cancer tissue was 13.76 but in normal tissue was 2.42 . The current study showed decreased in PTEN expression in breast tumor tissue in only $16 \%$ of patients compared to expression in normal breast tissue, which was similar to Palimaru et al study (Palimaru et al., 2013), it has been reported that low of PTEN $m R N A$ expression in breast carcinoma tissue occurs in only $20 \%$ of patients compared to normal breast tissue. In a study at 2018 for measuring PTEN $m R N A$ expression in breast carcinoma tissue and compared to expression in normal breast tissue for 78 breast cancer patients, a reduced PTEN expression in breast carcinoma tissue was found in only $33.3 \%$ of patients (Salmani et al., 2018). The two previous studies used quantitative method by real time PCR for assessment PTEN $m R N A$ expression. Also, a reduced or absent of PTEN expression in breast cancer tissue was only in 28 out 85 of breast cancer patients $(33 \%)$ in the Engin et al., (2006) study, which was using IHC for the detection of PTEN. There were other studies did not correspond to our data in this study, and reported decreased or absent PTEN expression in breast carcinoma tissue in high percentage among the breast cancer patients (Kechagioglou et al., 2014; Gschwantler-Kaulich et al., 2017; Shabbir et al., 2017).

As listed in Table 2, PTEN expression did not have any relation to any clinicopathological factors $(\mathrm{P}>0.05)$, there were also some studies that showed no association between PTEN expression and clinicopathological factors of breast cancer. The study mentioned above reported significance relation between PTEN expression and lymphatic invasion only $(\mathrm{P}=0.046)$, and showed not association with others clinicopathological parameters (Salmani et al., 2018). Also, the Palimaru et al., (2013) study did not find correlation between PTEN expression and lymph node status or with any clinicopathological factors, but other studies reported presence relation between PTEN expression and some clinicopathological factors (Li et al., 2015; Golmohammadi et al., 2016; Wang et al., 2017). A study at 2017 conducted a meta-analysis of 27 studies which included 10,231 patients to assess the relations of PTEN expression with clinicopathological characteristics, the PTEN loss had relations with larger tumor size $(\mathrm{P}=0.0006)$, lymph node metastasis $(\mathrm{P}=0.0001)$, negative ER $(\mathrm{P}=0.03)$, and negative $\mathrm{PR}$ $(\mathrm{P}=0.02)$, and reported no significant relation between PTEN loss and HER2 status (Li et al., 2017).

PIK3CA plays important role in breast cancer treatment, the FDA on May 2019 approved alpelisib (Piqray tablets) to be used in combination with fulvestrant (endocrine therapy) for the treatment of postmenopausal women, and men, with $H R+, H E R 2-$, PIK3CA-mutated, 
advanced or metastatic breast cancer. The treatment decision by this drug depend on identification of $P I K 3 C A$ mutations, therefore the FDA also approved the therascreen PIK3CA Kit (PIK3CA biomarkers) for detection of activating mutations in the PIK3CA gene (ASA, 2019). PIK3CA gene mutations are the most common in $H R+/ H E R 2-B C$, which are estimated to be present in approximately $40 \%$ of $H R+/ H E R$ - advanced or metastatic BC patients (Tolaney et al., 2019).

According to our results, we encourage and recommend further studies include a large size number of breast cancer patients to further evaluate the relations of $P I K 3 C A$ and PTEN genes expressions to clinical pathological characteristics of breast cancer, and assess the PIK3CA and PTEN proteins expressions and compare with the mRNA expression of these genes.

In conclusion, the results of the current study showed higher significantly increased PIK3CA $m R N A$ expression in breast tumor tissue compared to normal breast tissue, and contrary to expected, we also observed increased PTEN expression in breast carcinoma tissue compared to normal breast tissue. The expressions of PIK3CA and $P T E N$ showed not significant relation with any clinicopathological characteristics of breast cancer, and reported not found correlation between PIK3CA and PTEN expressions.

\section{Acknowledgments}

\section{Ethics approval}

Written informed consent was obtained from each patient and the study was approved by the Ethical Committee of the Genetic Engineering and Biotechnology Research Institute, University of Sadat City.

\section{Funding}

The authors received no financial support for the research, authorship, or publication of this article.

\section{References}

Aleskandarany MA, Rakha EA, Ahmed MAH, et al (2010). PIK3CA expression in invasive breast cancer: a biomarker of poor prognosis. Breast Cancer Res Treat, 122, 45-53.

Arnedos M, Vicier C, Lefebvre C, et al (2015). Precision medicine for metastatic breast cancer-limitations and solutions. Nat Rev Clin Oncol, 12, 693-704.

Jones D (2019). Novartis: FDA approves Novartis Piqray ${ }^{\mathbb{B}}$ the first and only treatment specifically for patients with a PIK3CA mutation in HR+/HER2-advanced breast cancer.

Bellacosa A, Kumar CC, Di Cristofano A, Testa JR (2005). Activation of AKT kinases in cancer: implications for therapeutic targeting. Adv Cancer Res, 94, 29-86.

Boyault S, Drouet Y, Navarro C, et al (2012). Mutational characterization of individual breast tumors: TP53 and PI3K pathway genes are frequently and distinctively mutated in different subtypes. Breast Cancer Res Treat, 132, 29-39.

Engelman JA, Luo J, Cantley LC (2006). The evolution of phosphatidylinositol 3-kinases as regulators of growth and metabolism. Nat Rev Genet, 7, 606.

Engin H, Baltali E, Güler N, et al (2006). Expression of PTEN, cyclin D1, P27/KIP1 in invasive ductal carcinomas of the breast and correlation with clinicopathological parameters.
PIK3CA and PTEN Genes Expressions in Breast Cancer

Bulletin du cancer, 93, 21-26.

Fruman DA, Rommel C (2014). PI3K and cancer: lessons, challenges and opportunities. Nature reviews. Drug Discov, 13, 140-56.

Golmohammadi RM, Rakhshani H, Moslem AR, Pejhan A (2016). Prognostic role of PTEN gene expression in breast cancer patients from north-east Iran. Asian Pac J Cancer Prev, 17, 4527-31.

Gschwantler-Kaulich D, Tan YY, Fuchs EM, et al (2017). PTEN expression as a predictor for the response to trastuzumabbased therapy in Her-2 overexpressing metastatic breast cancer. PLoS One, 12, e0172911.

Ibrahim AS, Khaled HM, Mikhail NN, et al (2014). Cancer incidence in Egypt: results of the national population-based cancer registry program. J Cancer Epidemiol, 2014.

Kechagioglou P, Papi RM, Provatopoulou X, et al (2014). Tumor suppressor PTEN in breast cancer: Heterozygosity, mutations and protein expression. Anticancer Res, 34, 1387-1400.

Leevers SJ, Vanhaesebroeck B, Waterfield MD (1999). Signalling through phosphoinositide 3-kinases: the lipids take centre stage. Curr Opin Cell Biol, 11, 219-25.

Leslie, Nick R, Downes CP (2004). PTEN function: how normal cells control it and tumour cells lose it. Biochem J, 382, 1-11.

Levine DA, Bogomolniy F, Yee CJA, et al (2005). Frequent mutation of the PIK3CA gene in ovarian and breast cancers. Clin Cancer Res, 11, 2875-8.

Li S, Shen Y, Yang J, et al (2017). Loss of PTEN expression in breast cancer: Association with clinicopathological characteristics and prognosis. Oncotarget, 8, 32043-54.

Li X, Fu L, Liu M, Yu X, Wang Q (2015). Expression of PTEN, p53 and EGFR in the molecular subtypes of breast carcinoma and the correlation among them. J Cent South Univ Med Sci, 40, 973-8.

Majumder PK, Sellers WR (2005). Akt-regulated pathways in prostate cancer. Oncogene, 24, 7465-74.

Palimaru I, Brügmann A, Wium-Andersen MK, Nexo E, Sorensen BS (2013). Expression of PIK3CA, PTEN mRNA and PIK3CA mutations in primary breast cancer: association with lymph node metastases. Springerplus, 2, 464.

Patani N, Martin LA, Dowsett MJ (2013). Biomarkers for the clinical management of breast cancer: international perspective. Int $J$ Cancer, 133, 1-13.

Salmani H, Hosseini A, Azarnezhad A, Ahmad H (2018). PTEN and $\mathrm{p} 53$ gene expressions in breast cancer specimens and their clinicopathological significance. Middle East J Cancer, 9, 105-11.

Samuels Y, Wang Z, Bardelli A, et al (2004). High frequency of mutations of the PIK3CA gene in human cancers. Science, 304, 554.

Bray F, Ferlay J, Soerjomataram I, et al (2018). Global cancer statistics 2018: GLOBOCAN estimates of incidence and mortality worldwide for 36 cancers in 185 countries. $C A$ Cancer J Clin, 68, 394-424.

Shabbir AM, Bin Ali Jerah A, Mohammad J, et al (2017). Promoter methylation and loss of expression of PTEN Gene in breast cancer patients from Saudi population. J Clin Exp Oncol, 6, 2.

Siegel RL, Miller KD, Jemal A (2016). Cancer statistics, 2016. CA Cancer J Clin, 66, 7-30.

Song MS, Salmena L, Pandolfi PP (2012). The functions and regulation of the PTEN tumour suppressor. Nat Rev Mol Cell Biol, 13, 283-96.

Tolaney S, Toi M, Neven GA (2019). Presented at: 2019 American Association for Cancer Research (AACR). Annual Meeting; March 29-April 3, 2019.

Tsutsui S, Inoue H, Yasuda K, et al (2005). Reduced expression 
Shaden Muawia Hanafy et al

of PTEN protein and its prognostic implications in invasive ductal carcinoma of the breast. Oncology, 68, 398-404.

Vanden Bempt I, Vanhentenrijk V, Drijkoningen M, et al (2005).

Real-time reverse transcription-PCR and fluorescence in-situ hybridization are complementary to understand the mechanisms involved in HER-2/neu overexpression in human breast carcinomas. Histopathology, 46, 431-41

Wang LL, Hao S, Zhang S, et al (2017). PTEN/PI3K/AKT protein expression is related to clinicopathological features and prognosis in breast cancer with axillary lymph node metastases. Hum Pathol, 61, 49-57.

Wang LQ, Zhang Q, Zhang JS, et al (2011). PI3K pathway activation results in low efficacy of both trastuzumab and lapatinib. BMC Cancer, 11, 1-10.

\section{c) (i) (8)}

This work is licensed under a Creative Commons AttributionNon Commercial 4.0 International License. 